\title{
The nasal mucosal late allergic reaction to grass pollen involves type 2 inflammation (IL-5 and IL-13), the inflammasome $(\mathrm{IL}-1 \beta)$, and complement
}

\author{
BR Leaker ${ }^{1,7}$, VA Malkov ${ }^{2,7}$, R Mogg $^{2,6}$, MK Ruddy $^{2,6}$, GC Nicholson ${ }^{1}$, AJ Tan ${ }^{3}$, C Tribouley ${ }^{2,6}$, G Chen $^{2,6}$,
} I De Lepeleire ${ }^{4}$, NA Calder ${ }^{4,6}, \mathrm{H} \mathrm{Chung}^{6}$, P Lavender ${ }^{5}$, LN Carayannopoulos ${ }^{2,6}$ and TT Hansel ${ }^{3}$

Non-invasive mucosal sampling (nasosorption and nasal curettage) was used following nasal allergen challenge with grass pollen in subjects with allergic rhinitis, in order to define the molecular basis of the late allergic reaction (LAR). It was found that the nasal LAR to grass pollen involves parallel changes in pathways of type 2 inflammation (IL-4, IL-5 and IL-13), inflammasome-related (IL-1ß), and complement and circadian-associated genes. A grass pollen nasal spray was given to subjects with hay fever followed by serial sampling, in which cytokines and chemokines were measured in absorbed nasal mucosal lining fluid, and global gene expression (transcriptomics) assessed in nasal mucosal curettage

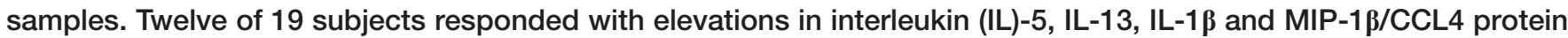
levels in the late phase. In addition, in these individuals whole-genome expression profiling showed upregulation of type 2 inflammation involving eosinophils and IL-4, IL-5 and IL-13; neutrophil recruitment with IL-1 $\alpha$ and IL-1 $\beta$; the alternative pathway of complement (factor $\mathrm{P}$ and $\mathrm{C} 5 \mathrm{aR}$ ); and prominent effects on circadian-associated transcription regulators. Baseline IL-33 mRNA strongly correlated with these late-phase responses, whereas a single oral dose of prednisone dose-dependently reversed most nasal allergen challenge-induced cytokine and transcript responses. This study shows that the LAR to grass pollen involves a range of inflammatory pathways and suggests potential new biomarkers and therapeutic targets. Furthermore, the marked variation in mucosal inflammatory events between different patients suggests that in the future precision mucosal sampling may enable rational specific therapy.

\section{INTRODUCTION}

Asthma and allergic rhinitis (AR) are increasing in prevalence in industrialized societies due to complex gene-environmental and microbial influences. ${ }^{1}$ Both asthma and AR are inflammatory disorders of the airways in which similar cell types and molecular pathways participate. ${ }^{2,3}$ Furthermore, nasal and bronchial disease co-exist in many asthma patients, with similar immunopathology, underlying the "one airway" hypothesis. ${ }^{4}$

Mast cells are the main effector cells of acute/early allergic responses, through allergen cross-linking-specific IgE molecules on the mast cell surface, and subsequent triggering of mast cell degranulation and release of vasoactive mediators. ${ }^{5}$ Eosinophils are involved in acute and chronic mucosal inflammation and migrate into the nasal and bronchial mucosa during the late allergic reaction (LAR). Allergic inflammation is frequently described as "type 2 inflammation" and has a characteristic gene expression signature in bronchial epithelium ${ }^{6}$ and sputum of asthmatics. ${ }^{7}$ IL- 5 and IL-13 mediate many of the features of type 2 inflammation, being produced by T-helper type-2 (Th2) cells and innate lymphoid cells of type 2 (ILC2 cells). ${ }^{8}$

Access to nasal mucosal secretions and cells is required to understand biomarkers and molecular mechanisms associated

${ }^{1}$ Respiratory Clinical Trials Ltd, London, UK. ${ }^{2}$ Merck Research Laboratories, Rahway, New Jersey, USA. ${ }^{3}$ Imperial Clinical Respiratory Research Unit (ICRRU), St Mary's Hospital, Imperial College, London, UK. ${ }^{4} \mathrm{MSD}$ (Europe) Inc., Brussels, Belgium. ${ }^{5}$ Department of Asthma, Allergy and Respiratory Science, King's College, London, UK and ${ }^{6}$ Present address: Celgene (L.N.C. and G.C.); Janssen R \& D, Spring House, PA (R.M.); Alnylam (M.K.R.); Novartis (C.T.); GSK (N.A.C.); Otsuka (H.C.). Correspondence: TT Hansel (t.hansel@imperial.ac.uk)

${ }^{7}$ The first two authors contributed equally to this work.

Received 13 April 2016; accepted 21 July 2016; published online 28 September 2016. doi:10.1038/mi.2016.74 
with AR. Either lavage or nasosorption using strips of absorptive paper have been used to sample nasal mucosal lining fluid (MLF) mediators. ${ }^{9-11}$ More recently, the nasosorption method has been refined by the use of synthetic materials and, as the approach is non-invasive, it has proved ideal for sampling from children with $\mathrm{AR}^{12}$ and infants. ${ }^{13}$ Serial nasosorption samples have been used after nasal allergen challenge (NAC), ${ }^{14}$ including to assess the effects of a monoclonal antibody against anti-IL-13. ${ }^{15}$ Nasal curettage is a technique to obtain nasal epithelial cells without local anesthesia and has been used for gene expression profiling after human rhinovirus infection, ${ }^{16}$ whereas nasal brushings have been used to define phenotypes of childhood asthma. $^{17}$

Detailed insights into molecular and cellular events in the airway mucosa are needed to define targets for new therapies, provide biomarkers for clinical trials, and to enable personalized medicine. ${ }^{2,18}$ This study examined the nasal mucosal LAR by measurement of cytokines and chemokines in MLF, and assessment of gene expression patterns in nasal mucosal specimens. We used non-invasive mucosal sampling, obtaining serial nasosorption samples and nasal epithelial curettage sample at baseline and $8 \mathrm{~h}$ after NAC. We compared the effects of single doses of oral prednisone with placebo on biomarkers of mucosal inflammation after NAC. In this manner, we documented molecular events of the nasal mucosal LAR and the effects of corticosteroids within individual subjects.

\section{RESULTS}

\section{Subjects and symptoms}

Nineteen subjects with a confirmed history of seasonal AR and skin prick-positive allergy to grass pollen were recruited and studied outside the London grass pollen season (Figure 1, Supplementary Table E1 online, and Supplementary Figure E1). There were no significant adverse events and all subjects completed the study. NAC induced a prominent rise in early $(1 \mathrm{~h})$ mean total nasal symptom scores (TNSS) with a diminution of symptoms into the late phase (2-8 h; Supplementary Figure E2). Participants were observed by clinical research staff to have allergic symptoms immediately after NAC, although the first TNSS after NAC were formally assessed at $1 \mathrm{~h}$. Symptoms consisted of sneezing for a few minutes, nasal discharge, and nasal and soft palate itching. Symptoms in the late phase ( $2-8 \mathrm{~h}$ post NAC) are subjective, variable, and are of low magnitude: in our study, reaching mean levels of $\sim 2$ out of 12 and being generally due to nasal obstruction. However, there were nonsignificant trends for IL13 Responders (see definition in Methods section-Statistical Analyses) to have higher early- $(1 \mathrm{~h})$ and late-phase (2-8h) TNSS (Supplementary Figures E2 and E3, with individual responses shown in Supplementary Figure E4). Prednisone also showed a nonsignificant trend for decreased late-phase $(2-8 \mathrm{~h})$ TNSS in Responders, when compared with placebo TNSS. TNSS in the late phase $(2-8 \mathrm{~h})$ correlated with those in the early phase $(0-1 \mathrm{~h})$ in the placebo arm followed by NAC $(r=0.774, P=1.01 \mathrm{E}-04)$ and in the prednisone at $10 \mathrm{mg}$ arm $(r=0.622, P=4.49 \mathrm{E}-03)$, whereas approaching statistical significance in the prednisone at $25 \mathrm{mg}$ arm $(r=0.449$, $P=0.0536)$. However, blood eosinophil counts, age and gender did not predict the late-phase TNSS $(2-8 \mathrm{~h})$ nor responder status.

\section{Nasal MLF cytokines and chemokines}

Overall, grass pollen NAC resulted in increases in type 2 cytokines (IL-5 and IL-13) and innate-factors (CCL4/MIP-1 $\beta$ and IL-1 $\beta$ ) from 2 to $8 \mathrm{~h}$, comprising a late-phase cytokine/ chemokine response (Figure 2a, Supplementary Figure E5, and Supplementary Tables E2-E4). Single-dose pretreatment with prednisone caused large and statistically significant dosedependent reduction of NAC-induced IL-5, IL-13, IL-1 $\beta$ and CCL4 compared with placebo (Figure 2a). The degree of correlation ( $>0.9$ ) between IL-5 and IL-13 fold changes under the different treatment regimens was remarkably high (Figure 2b). Inspection of individual patient IL-5 and IL-13 responses revealed significant between-subject variation that was used as the basis for defining responders (see MethodsStatistical Analyses) (Figure 2c and Supplementary Figure E6). IL-1 $\beta$ is present in unchallenged noses and levels increase slightly following NAC. However, corticosteroid given before NAC causes an apparent decrease in levels of IL-1 $\beta$, which we speculate may be due to the effects of steroids and repeated sampling.

\section{mRNA profiling}

Prespecified analyses of mRNAs and cell-type-associated signatures responsive to allergen and prednisone. Initial prespecified analysis of all participants' nasal mRNA using an absolute foldchange threshold of 1.5 and a Benjamini-Hochberg false discovery rate (FDR) set at $\leq 10 \%$, yielded 2,693 and 136 probesets responsive to allergen and $25 \mathrm{mg}$ prednisone, respectively (Supplementary Datafile S1). Among transcripts strongly increased by NAC, those of immunologic receptors and secreted products were among the most frequent. This included those associated with the expected allergen-activated type 2 inflammation elements such as eosinophils and Th2 cells (e.g., CLC/galectin-10, HRH/histamine receptor 4, CCR3/ eotaxin receptor, CCL23, and CD69), as well as those associated with broader immune/inflammatory responses and innate immune effectors (e.g., IL1A, IL1B, OSM/oncostatin M, CCL2, TGFB2, CD300, CLEC4A, and CD1C). In contrast, mRNAs with decreased levels following NAC include those of proteins participating in circadian-related signaling (e.g., PER1, PER3, NR1D1/REV-ERB $\alpha$, and C1orf51/CIART) and other non-immune functions including epithelial growth and structure (e.g., MCAM/CD146, NRCAM, CYP1B1, LCE3D, and LAMB1/laminin $\beta 1$ ), and cell division (e.g., NUSAP1/ nucleotide and spindle associated protein, CDC25B, CDCA7, and SKP2). In general, prednisone pretreatment led to suppression of NAC-induced changes, with a few exceptions such as TGFB2. Figure 3a illustrates the strongly opposing effects of allergen and prednisone that were observed. 
a

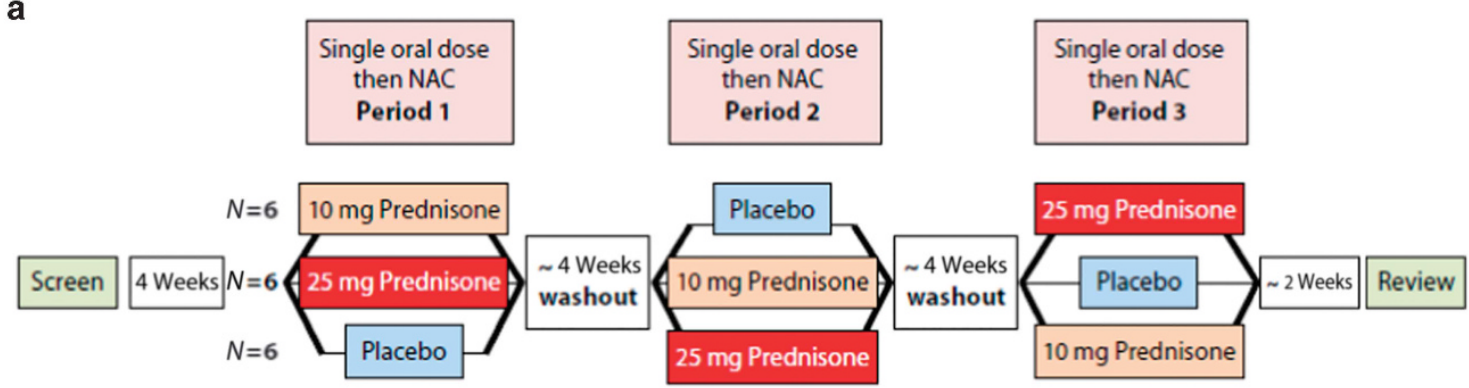

b

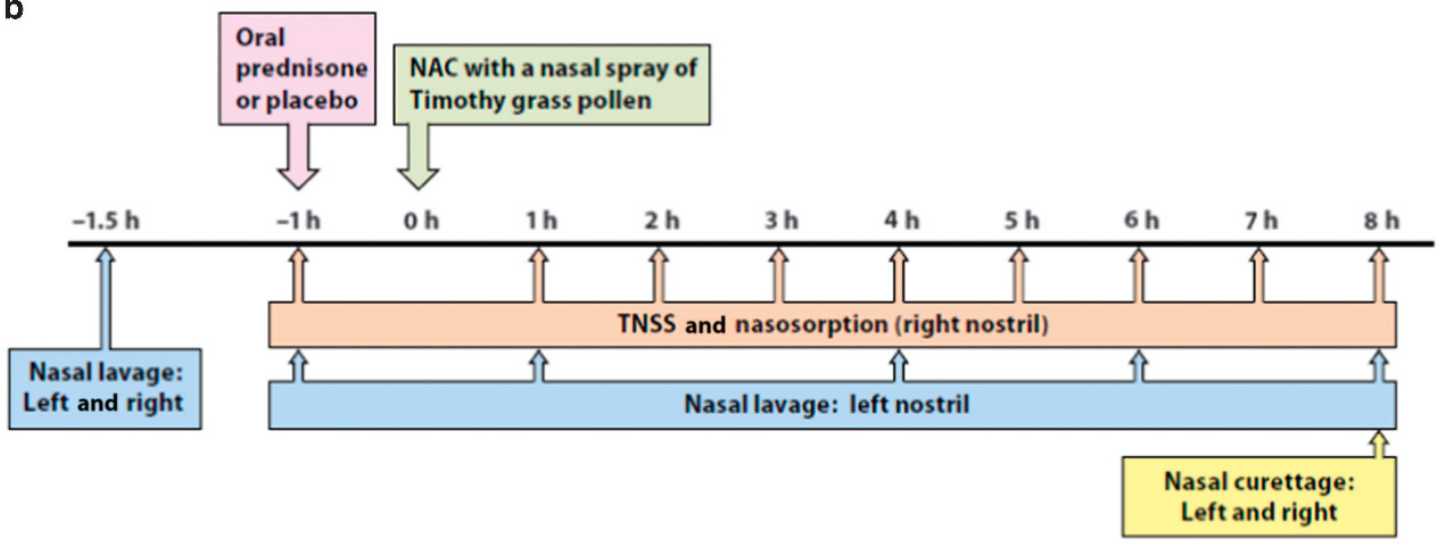

C
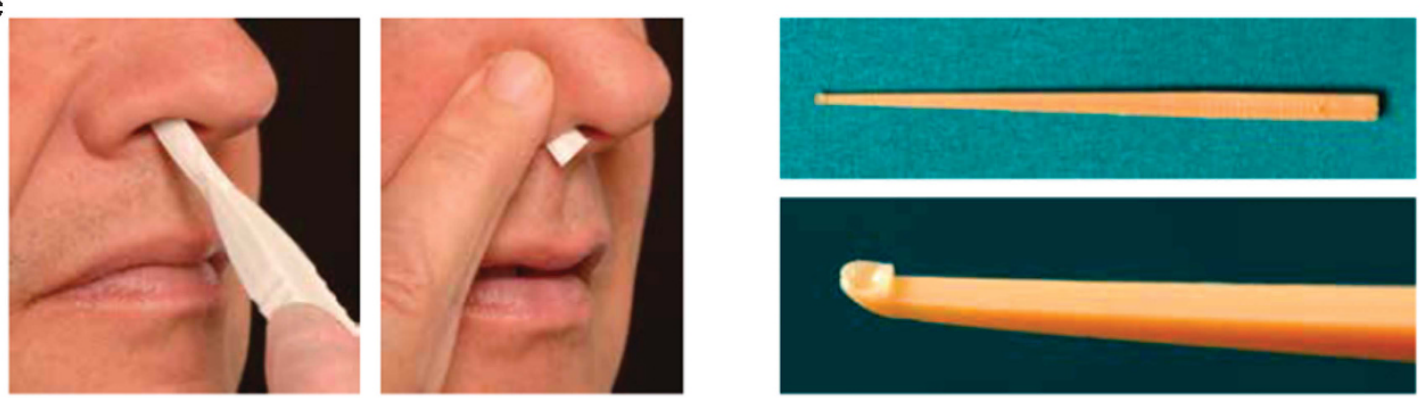

Nasosorption:

SAM for MLF soluble inflammatory mediators

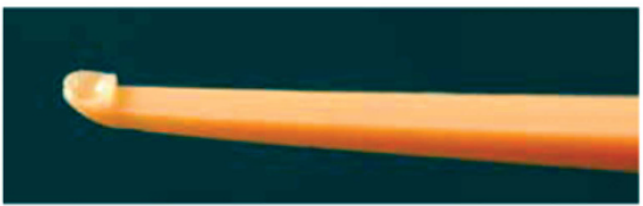

Nasal mucosal curettage:

Rhinoprobe sample for transcriptomics at $8 \mathrm{~h}$ post NAC

Figure 1 Study design and nasal mucosal sampling procedures. (a) Randomized, double-blind, three-way crossover study of the effects of a single oral dose of prednisone (10 or $25 \mathrm{mg}$ ) versus placebo given $1 \mathrm{~h}$ before nasal allergen challenge (NAC). (b) Events taking place during NAC study visits. Both nostrils were lavaged and the oral tablet given (prednisone or placebo). NAC was performed with Timothy grass pollen extract (nasal spray of $100 \mu \mathrm{l}$, with $\mathrm{Phl} \mathrm{p} 5$ allergen at $1 \mu \mathrm{g}$ per nostril. Total nasal symptom scores and nasosorption sampling of the right nostril were carried out at hourly intervals to $8 \mathrm{~h}$ with lavages at 1, 4, 6, and $8 \mathrm{~h}$ post NAC on the left nostril and finally bilateral nasal curettage with a Rhinoprobe at $8 \mathrm{~h}$. (c) Nasal sampling techniques consisted of nasosorption with synthetic absorptive matrix (SAM) and nasal curettage with a $10 \mathrm{~cm}$-long plastic curette (screening and $8 \mathrm{~h}$ post NAC).

In addition to individual mRNA levels, the expression of prespecified multigene composite scores associated with eosinophils, neutrophils, and mast cells was also measured (Supplementary Table E5). As shown in Figure $\mathbf{3 b}$, eosinophil- and neutrophil-related composite scores showed strong post-NAC average induction and suppression by prednisone pretreatment.

Patterns of inter-individual gene expression: responders and nonresponders. In order to identify natural patterns in NACresponse expression data, two unsupervised approaches were applied. In the first, hierarchical clustering of the 365 probe sets strongly responsive to allergen (Supplementary Datafile S1) was performed using correlations between changes in expression ( $8 \mathrm{~h}$ after NAC versus study baseline) as the distance metric. In this analysis, IL-13 responders were more likely to be similar in terms of gene expression, whereas non-responders had distinct and more heterogeneous patterns (Supplementary Figure E7). To confirm this finding, a second approachprincipal components analysis-was applied. The first two principal components, encompassing $55 \%$ of the total variance, sufficed to separate IL-13 responders from non-responders (Supplementary Figure E8). Indeed, the first principal component alone, encompassing $\sim 41 \%$ of the total 


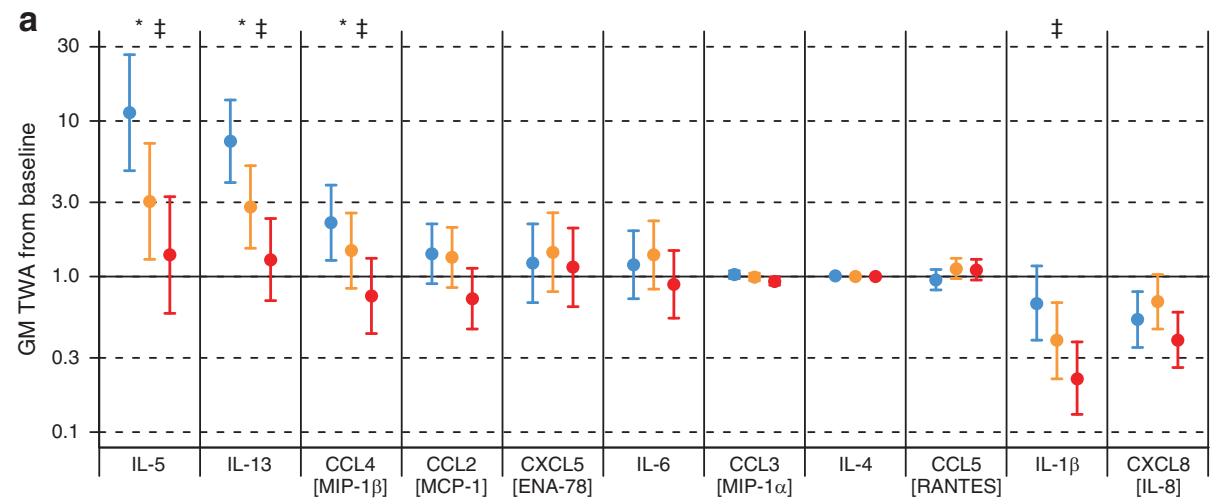

Cytokine or chemokine
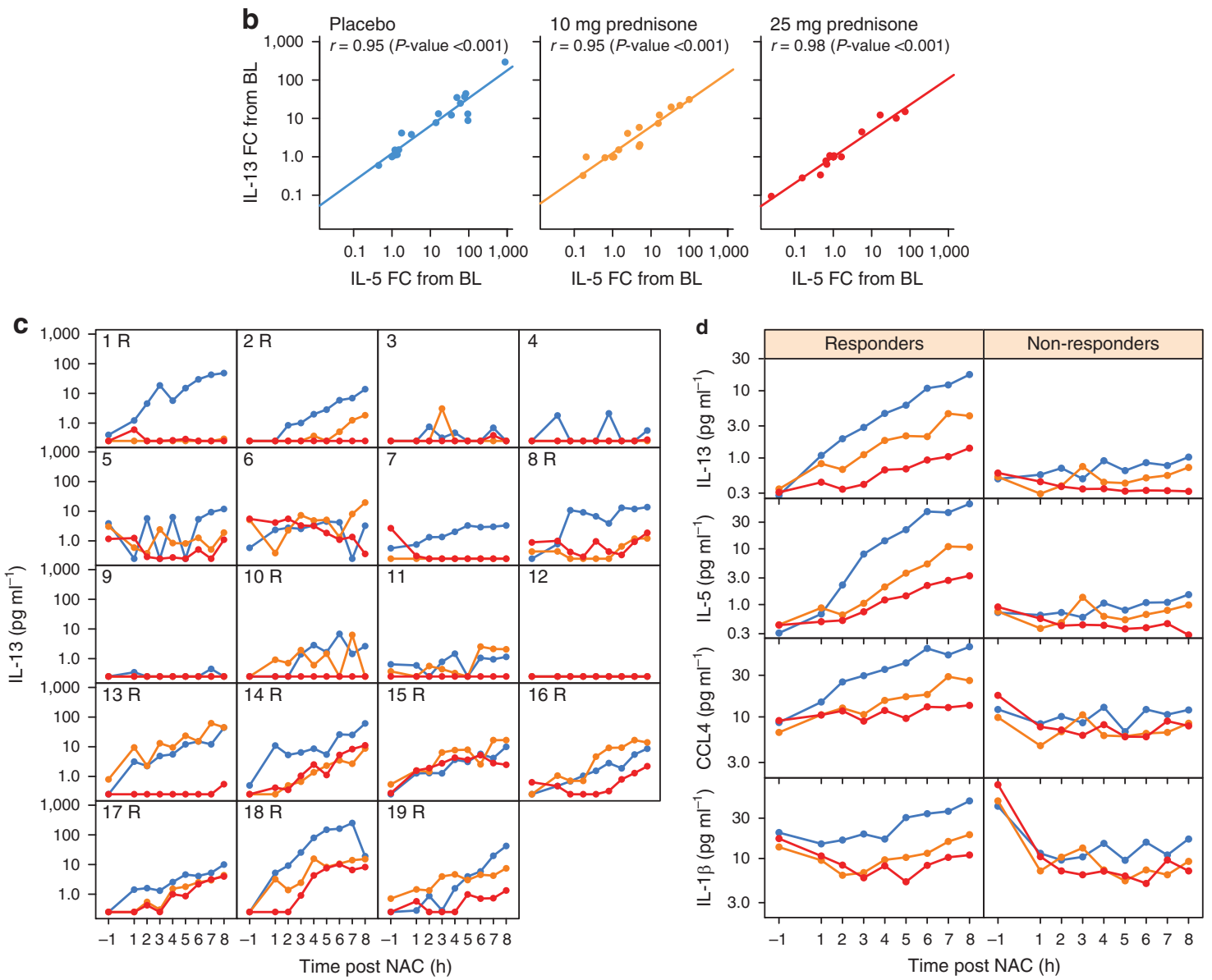

Figure 2 Cytokine and chemokine levels in nasal mucosal lining fluid (MLF). (a) Geometric mean 2-8 h time-weighted average (GMTWA change from baseline in cytokine or chemokine levels in response to NAC. Error bars represent $90 \% \mathrm{Cl}$, but are not shown in $\mathbf{d}$ for clarity. Asterisks signify allergen responses (placebo pretreatment before NAC) with $P<0.05$. Double daggers signify dose-responsive effects (versus placebo) of prednisone pretreatment with $P<0.05$ for all comparisons. (b) Dot plot of IL-13 versus IL-5 fold changes from baseline under each treatment. Pearson's correlations appear in each panel. Solid lines represent least-squares regression lines. (c) Time courses of IL-13 production in individual participants. "R" refers to an IL-13 responder. (d) Time courses of geometric mean cytokine levels in IL-13 responders (left panels) and non-responders (right panels). $N=18$ subjects (one subject yielded insufficient nasal lining fluid for full analysis). For all panels, blue, orange, and red symbols signify placebo, $10 \mathrm{mg}$ prednisone, and $25 \mathrm{mg}$ prednisone pretreatment, respectively. BL, baseline; $\mathrm{Cl}$, confidence interval; FC, fold change; GMTWA, geometric mean time-weighted average; IL, interleukin, NAC, nasal allergen challenge.

variance, not only separates nasal type- 2 cytokine responders from non-responders but also correlates strongly $(r=\sim 0.8$, Figure 4a) with GMTWA ${ }_{2-8 \mathrm{~h}}$ (geometric mean time-weighted average cytokine concentration measured by immunoassay at $2-8 \mathrm{~h}$ ) of type- 2 cytokine secretion, suggesting commonality of the biology underlying IL-13 production and mRNA content changes following NAC.

Baseline gene expression and the IL-13 response. Baseline gene expression was measured during the screening period, which 
a

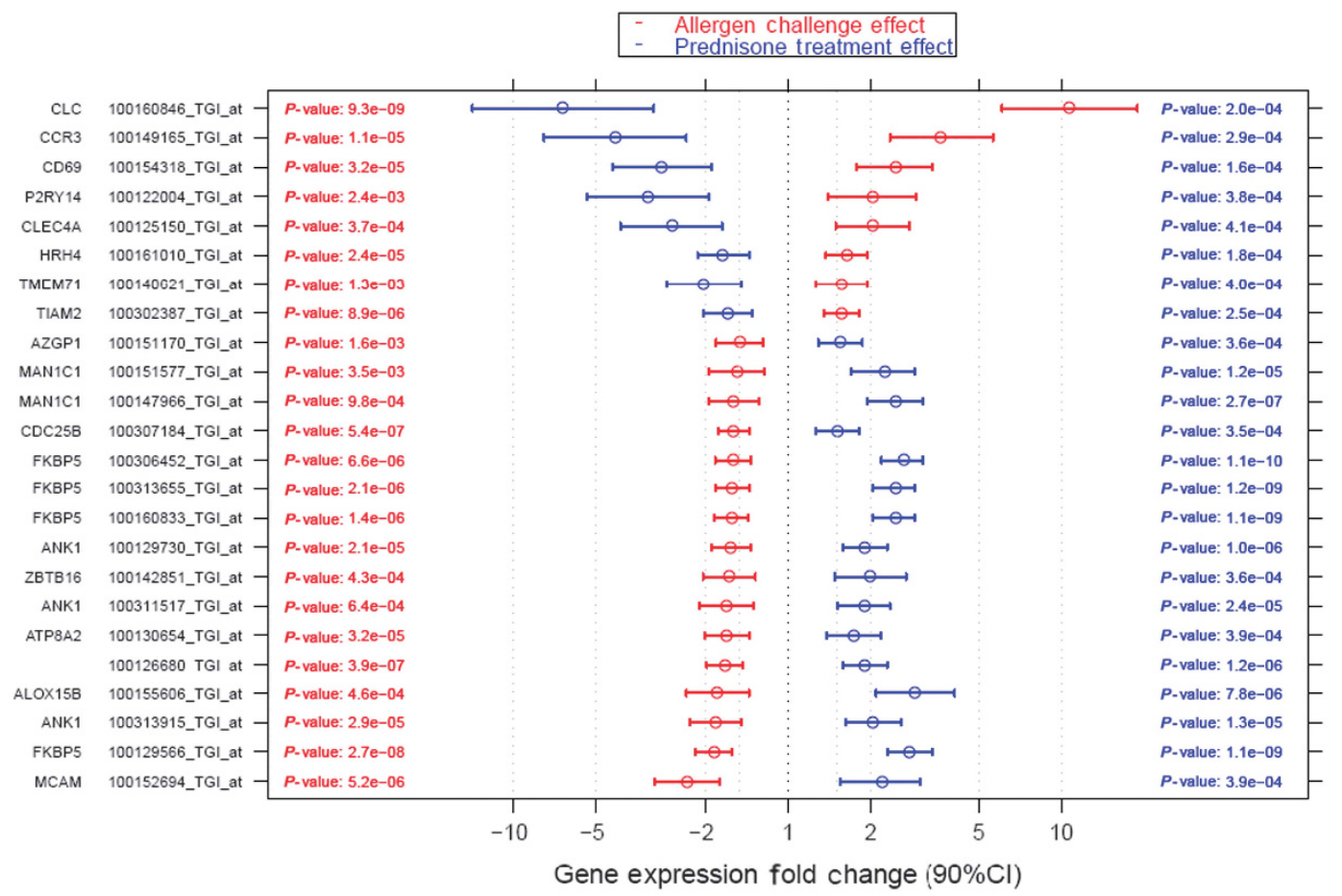

b
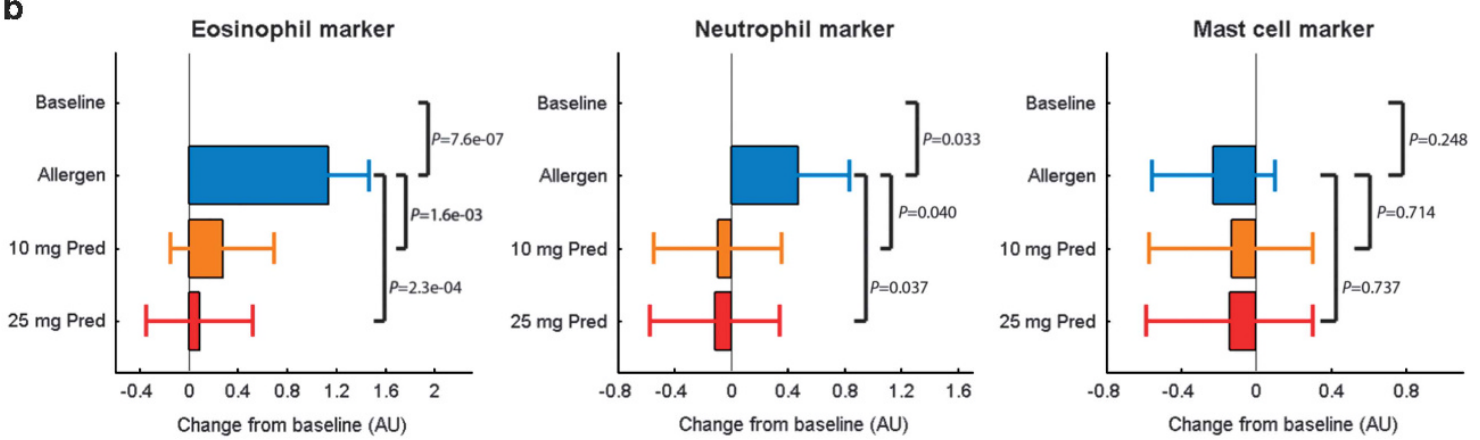

Figure 3 Gene expression (mRNA) changes in response to nasal allergen challenge (NAC) and prednisone pretreatment. (a) Fold changes in probesets responsive to allergen (placebo pretreatment) and $25 \mathrm{mg}$ prednisone. All subjects' RNA samples were studied and probesets having absolute fold change $>1.5$ and false discovery rate (FDR) $<10 \%$ are presented. Red symbols and $P$-values represent allergen effects (placebo pretreatment), whereas blue symbols and $P$-values represent effects of $25 \mathrm{mg}$ prednisone versus placebo. (b) Changes in cell-type-associated mRNA signatures $8 \mathrm{~h}$ after allergen challenge, with and without prednisone pretreatments. (b) $P$-values are uncorrected for multiplicity.

was 1-3 months before subjects were given placebo and then subsequent NAC; the time gap across subjects differed based on randomization and exact period when placebo was given. This baseline expression was examined to determine whether genes associated with asthma might also relate to the post-NAC IL-13 responses. Published GWAS has identified a number of genetic modifiers of asthma: CHI3L1, IL6R, DENND1B, IL1RL1, IL18R1, PDE4D, RAD50, IL13, IL33, SMAD3, ORMDL3, GSDMB, IL2RB, and PYHIN1. ${ }^{19}$ Accordingly, correlations of these gene transcript levels with IL-13 and IL-5 cytokine responses was measured. Of these correlations, only that between baseline IL33 mRNA and NAC IL-13/IL-5 response had Bonferroni-corrected significance below 0.05 (Figure $4 \mathrm{~b}$ and Supplementary Table E6). NAC late-phase responders tended to have higher levels of prechallenge IL33 nasal mucosal mRNA than did non-responders.
The level of baseline IL33 mRNA expression showed a strong correlation with the magnitude of type- 2 cytokine induction following NAC ( $r \sim 0.7, P<0.01$, Figure $4 \mathbf{b})$, suggesting a mechanistic basis for variation in magnitude of type-2 cytokine responses to allergen.

Further analysis was carried out for potential de novo gene signatures at baseline with a reasonable FDR (i.e., $<0.2)$ that would predict subsequent NAC Responders (Supplementary Table E7). However, the study sample size was based on the primary endpoint and was too small to detect baseline prediction gene signatures.

A correlation matrix and dendrogram (Supplementary Figure E9) demonstrated that Responder status is associated with certain parameters measured after NAC: IL-13 and IL-5 protein, the first principal component across the 
a

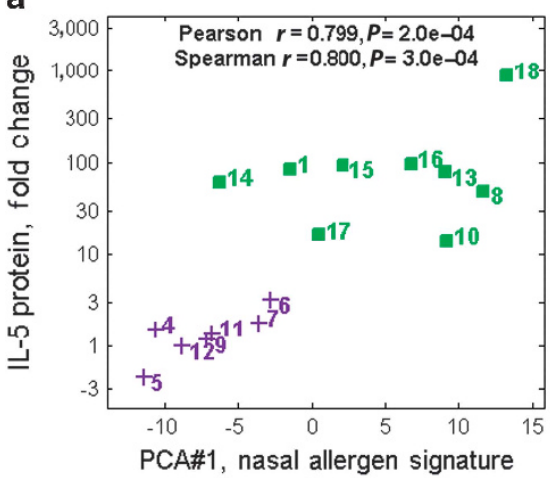

b

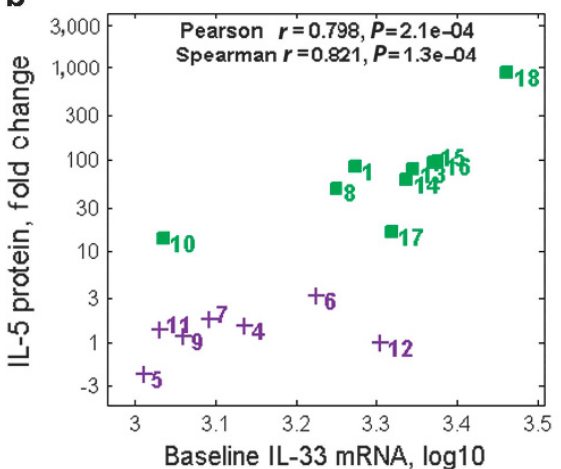

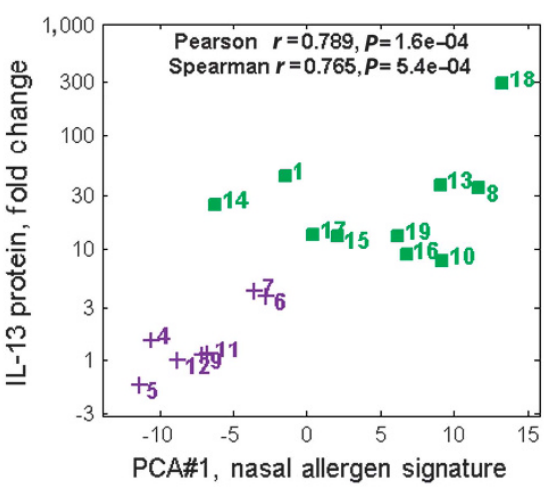

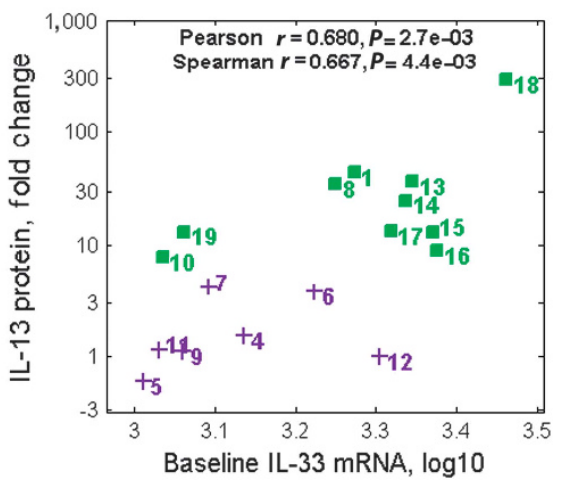

Figure 4 Gene expression (mRNA) changes in nasal curettage samples with respect to interleukin (IL)-5 and IL-13 protein levels. Dot plots of allergeninduced GMTWA $\mathrm{A}_{2-8 \mathrm{~h}}$ (geometric mean time-weighted average cytokine concentration measured by immunoassay at 2-8 h) log fold change from baseline in nasal lining fluid IL-5 and IL-13 compared with (a) first principal component coefficient of nasal scrape gene expression for subjects pretreated with placebo and (b) baseline nasal scrape IL-33 mRNA expression. Green squares and purple cross characters correspond to cytokine responders and nonresponders, respectively. Pearson's and Spearman's correlation coefficients for the relationships appear above the respective dot plots.

allergen gene expression signature, the eosinophil gene signature, and gene expression for CCL23, IL-4, IL-13, and CD69. There is weaker correlation with a cluster for the neutrophil gene expression signature, IL1A and IL1B. The Responder group had only weak correlation with TNSS (2-8 h). Importantly, as expected, given its strong correlation with IL-5 and IL-13 levels, IL33 gene expression at baseline correlated with post-NAC IL-13 Responder status, (Supplementary Figure E10). In addition, TNSS at $2-8 \mathrm{~h}$ did not correlate with IL-5 and IL-13 protein or mRNA expression (Supplementary Figure E11).

Pathways of inflammation genes and circadian-associated genes. Ingenuity Analysis was performed in IL-13 responders, with 1,214 genes having an FDR of $<0.05$, of which 41 genes were selected on the basis of their immune function (being cytokines, chemokines, soluble immunoregulatory factors, and their receptors) and being involved in circadian-associated transcription regulation (Figure 5). Ingenuity Pathway Analysis was applied to the mRNA profiling data using the Core Analysis tool to identify canonical pathways enriched with genes determined to be differentially expressed (Figure 5a).

Increased gene expression after NAC involves four main inflammatory pathways: type 2 inflammation (IL-4, IL-5,
IL-13, and CCR3), innate immunity mediated through IL- $1 \alpha$, IL-1 $\beta$, IFNAR1, and the inflammasome, and the alternative pathway of complement activation (factor P and C5AR1; Figure 5b). Significantly decreased gene expression was found for all five components of classical complement factor 1 (C1QA, C1QB, C1QC, C1R, and C1S). Gene expression for circadianassociated mRNA was particularly modulated by allergen challenge. Figure $5 c$ shows the effects of prednisone $25 \mathrm{mg}$ in NAC-induced changes: this is approximately the opposite of the NAC effect, demonstrating the potent and broad effect of corticosteroids on the allergic inflammatory response. Table 1 (responses averaged over responders) and Figure 6 (individual patient responses) provide quantitative views of these observations.

Individuals responding to NAC with IL-5 and IL-13 in nasal MLF, also showed increases in eosinophil-related, type 2-like, neutrophil-related, IL-1-related, and circadian-associated gene expression (Table 1 and Figure 6). Prednisone pretreatment, as in the averaged transcript effects shown in Figure 3a, clearly suppressed allergen effects at the individual level for the analytes shown in Figure 6b. However, in some patients the complement signal was not inhibited by corticosteroid. Circadian-associated genes were differentially regulated either up or down by NAC; notably, in some patients these changes were not opposed by corticosteroid. 


\begin{tabular}{|l|l|}
\hline \multicolumn{1}{|c|}{ Name } & $P$-value \\
\hline Airway inflammation in asthma & $1.43 \mathrm{E}-03$ \\
\hline Circadian rhythm signaling & $2.01 \mathrm{E}-03$ \\
\hline Complement system & $2.01 \mathrm{E}-03$ \\
\hline $\begin{array}{l}\text { Pattern recognition receptors (PRR) } \\
\text { for bacteria and viruses }\end{array}$ & $4.6 \mathrm{E}-03$ \\
\hline
\end{tabular}

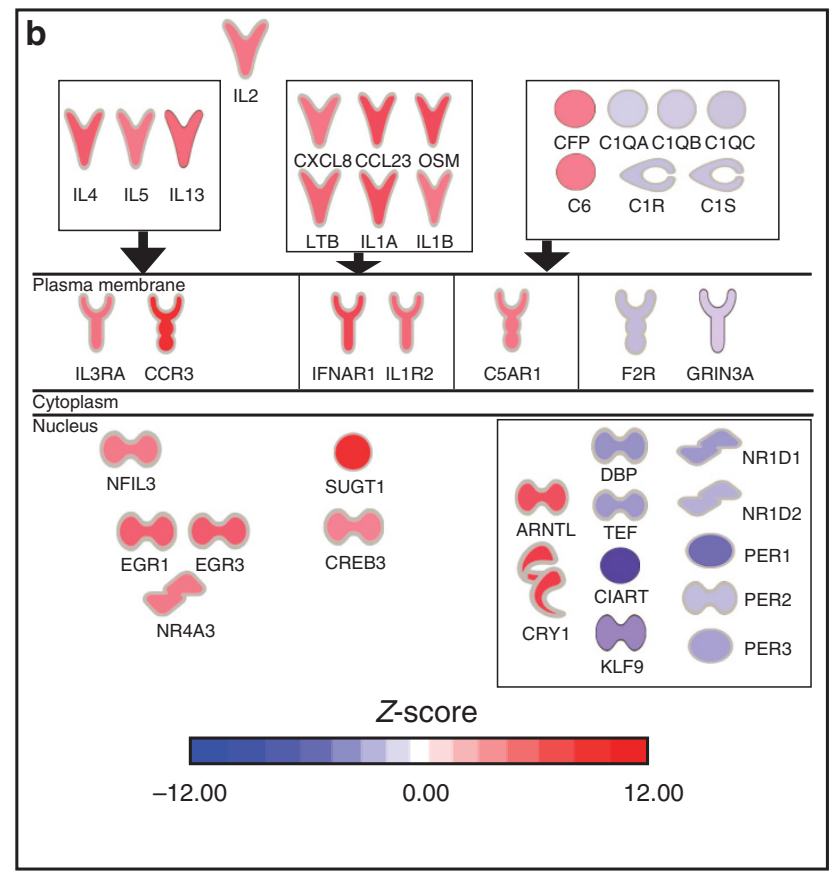

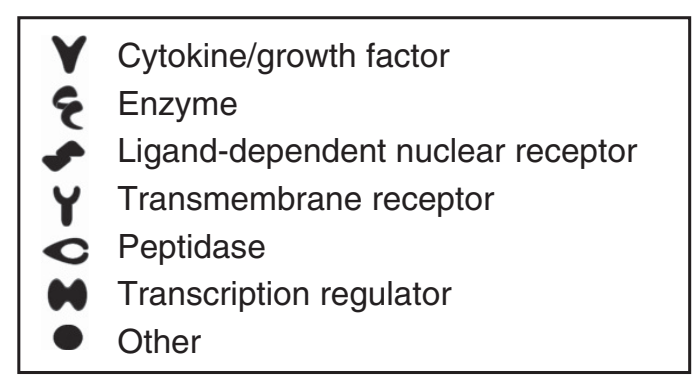

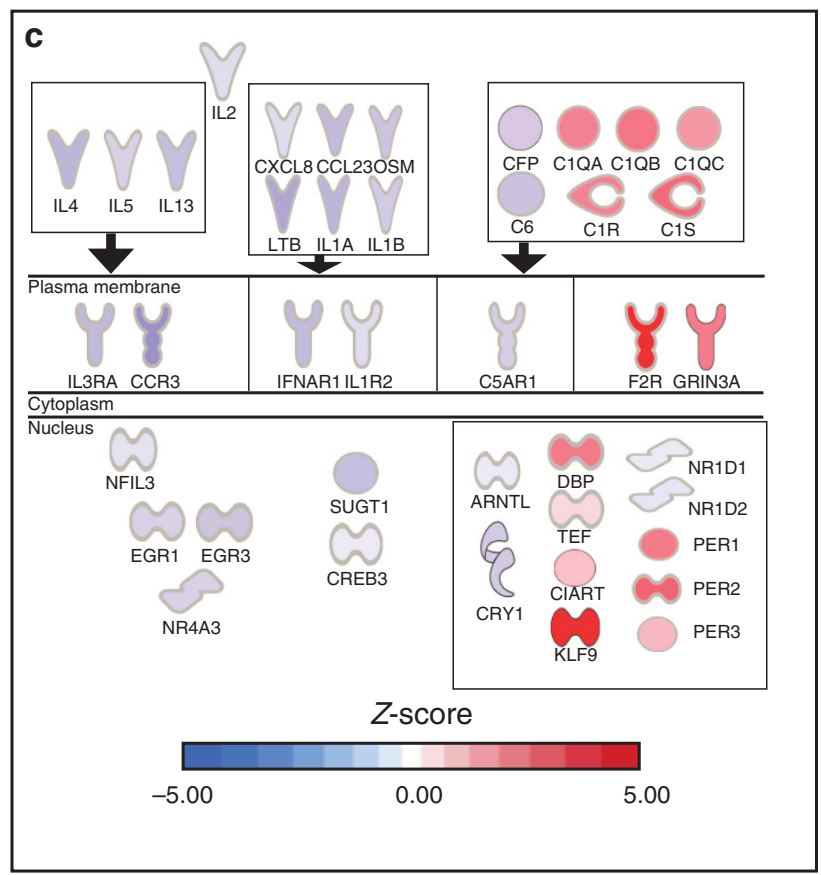

Figure 5 Pathways/gene groups highlighted by Ingenuity analysis of mRNA data in responders. (a) Ingenuity analysis in interleukin (IL)-13 responders (see Methods and Results for definition) to show top canonical pathways, within 1,214 genes having a false discovery rate (FDR) of $<0.05$. (b) Schematic to show selected gene expression changes with placebo then nasal allergen challenge (NAC) and curettage at $8 \mathrm{~h}$, compared with baseline gene expression. (c) Effects of a single dose of prednisone $(25 \mathrm{mg}$ orally) on gene expression changes with NAC and curettage at $8 \mathrm{~h}$, relative to post placebo NAC at $8 \mathrm{~h}$ versus baseline. Genes have been grouped according to type 2 inflammation cytokines (IL4, IL5, and IL13) and receptors (CCR3/eotaxin receptor and IL-3R), a mixed group of inflammatory cytokines (IL1 $\alpha$, IL-1 $\beta$, IL-2, IL-8, CCL23/MIP-3, OSM, and LTB/TNFC) and receptors (IFNAR1 and IL1R2), complement factors (CFP and C6) and their receptors (C5AR1), and circadian-associated transcription factors and controllers (BMAL-1 = ARNTL and CRY1). F2R is thrombin receptor and protease-activated receptor (PAR)-1, and links with the coagulation pathway. Certain complement factors (C1QA, C1QB, C1QC, C1R, and C1S) and circadian-associated transcription regulator genes (CIART (C1orf51), PER1-3, etc) were prominently downregulated by NAC. Red represents upregulation, whereas blue represents downregulation. Blue and red color intensity represents $Z$-score for the effect.

\section{DISCUSSION}

This study indicates that the nasal LAR involves gene and protein expression changes reflecting a set of inflammatory processes: type 2 eosinophilic inflammation associated with IL-4/5/13 release, inflammasome-type neutrophilic activation with IL- $1 \alpha / \beta$, upregulation of the alternative pathway of complement involving factors $\mathrm{P}$ and $\mathrm{D}$, and involvement of coagulation through the thrombin receptor (protease-activated receptor-1). Stimulation of innate immune responses by grass pollen is consistent with house dust mite allergen having been shown to functionally mimic a component of the Toll-like receptor 4 complex and causing an innate immune response. ${ }^{20}$
In addition, grass pollen Phleum P1 can directly stimulate IL-1/ $6 / 8$ production by airway epithelial cells, ${ }^{21}$ whereas whole grass extracts can stimulate bronchial epithelial cells to secrete cytokines. $^{22}$

Serial non-invasive sampling was carried out from the nasal mucosa to provide a range of biomarkers that reflect molecular features of the reaction to allergen. We demonstrated the utility of this approach in relation to a single oral dose of corticosteroid, establishing NAC in the context of serial precision mucosal sampling from an accessible nasal site. There was a focus on establishing the features of the IL- 5 and IL- 13 response by immunoassay of MLF and linking this response with gene expression profiling, and then identifying potential molecular 
Table 1 Altered gene expression for cytokines, chemokines, complement factors, and circadian-associated genes: NAC and the effects of prednisone

\begin{tabular}{|c|c|c|c|c|}
\hline Gene probe set & Notes & Pre-NAC treatment & Fold change (Cl 90\%) & P-value \\
\hline $\mathbb{I L}-4$ & Interleukin-4 & Allergen & $1.846(1.44,2.35)$ & 0.00022 \\
\hline \multirow[t]{2}{*}{ 100136820_TGl_at } & & 10 mg Prednisone & $0.653(0.47,0.9)$ & 0.03778 \\
\hline & & 25 mg Prednisone & $0.557(0.40,0.77)$ & 0.00624 \\
\hline IL-5 & Interleukin-5 & Allergen & $3.468(1.90,6.32)$ & 0.0016 \\
\hline \multirow[t]{2}{*}{ 100135525_TGl_at } & & 10 mg Prednisone & $0.568(0.25,1.25)$ & 0.23341 \\
\hline & & 25 mg Prednisone & $0.407(0.1,0.89)$ & 0.0649 \\
\hline IL-13 & Interleukin-13 & Allergen & $1.748(1.36,2.23)$ & 0.00069 \\
\hline \multirow[t]{2}{*}{ 100125222_TGI_at } & & 10 mg Prednisone & $0.711(0.52,0.96)$ & 0.06614 \\
\hline & & 25 mg Prednisone & $0.627(0.46,0.84)$ & 0.01519 \\
\hline CCR3 & Eotaxin-1/2/3 receptor & Allergen & $8.056(4.78,13.5)$ & $3.01 \mathrm{E}-7$ \\
\hline \multirow[t]{2}{*}{ 100149165_TGl_at } & & 10 mg Prednisone & $0.193(0.09,0.39)$ & 0.00064 \\
\hline & & 25 mg Prednisone & $0.154(0.07,0.31)$ & 0.00015 \\
\hline$I L-1 \alpha$ & Interleukin-1 $\alpha$ & Allergen & $5.9(2.80,12.5)$ & 0.00042 \\
\hline \multirow[t]{2}{*}{ 100163104_TGI_at } & & 10 mg Prednisone & $0.351(0.12,0.97)$ & 0.09366 \\
\hline & & 25 mg Prednisone & $0.265(0.0,0.73)$ & 0.03652 \\
\hline$I L-1 \beta$ & Interleukin-1 $\beta$ & Allergen & $10.69(4.55,25.1)$ & 0.00007 \\
\hline \multirow[t]{2}{*}{ 100126016_TGl_at } & & 10 mg Prednisone & $0.212(0.06,0.68)$ & 0.03322 \\
\hline & & 25mg Prednisone & $0.128(0.04,0.40)$ & 0.00655 \\
\hline OSM & Oncostatin-M & Allergen & $6.627(3.42,12.8)$ & 0.00005 \\
\hline \multirow[t]{2}{*}{ 100137287_TGI_at } & & 10 mg Prednisone & $0.256(0.10,0.64)$ & 0.01779 \\
\hline & & 25 mg Prednisone & $0.263(0.1,0.65)$ & 0.01932 \\
\hline IFNAR1 & IFN- $\alpha$ receptor & Allergen & $1.352(1.22,1.49)$ & 0.00003 \\
\hline \multirow[t]{2}{*}{ 100303602_TGl_at } & & 10 mg Prednisone & $0.824(0.71,0.94)$ & 0.02721 \\
\hline & & 25 mg Prednisone & $0.79(0.68,0.91)$ & 0.00894 \\
\hline C5AR1 & Complement C5a receptor-1 & Allergen & $1.968(1.43,2.0)$ & 0.00118 \\
\hline \multirow[t]{2}{*}{ 100302987_TGl_at } & & 10 mg Prednisone & $0.562(0.36,0.87)$ & 0.03555 \\
\hline & & 25mg Prednisone & $0.581(0.37,0.90)$ & 0.04556 \\
\hline CFP & Complement factor properdin & Allergen & $2.269(1.57,3.27)$ & 0.00075 \\
\hline \multirow[t]{2}{*}{ 100145758_TGI_at } & & 10 mg Prednisone & $0.38(0.2,0.64)$ & 0.00367 \\
\hline & & 25 mg Prednisone & $0.354(0.21,0.58)$ & 0.00171 \\
\hline C1R & Complement 1R Subcomponent & Allergen & $0.487(0.36,0.65)$ & 0.00034 \\
\hline \multirow[t]{2}{*}{ 100145579_TGl_at } & & 10 mg Prednisone & $1.682(1.11,2.54)$ & 0.0408 \\
\hline & & 25 mg Prednisone & $1.557(1.03,2.34)$ & 0.07687 \\
\hline C1QA & Complement 1Q A chain & Allergen & $0.577(0.45,0.72)$ & 0.00041 \\
\hline \multirow[t]{2}{*}{ 100159021_TGl_at } & & 10 mg Prednisone & $1.438(1.04,1.98)$ & 0.06352 \\
\hline & & 25 mg Prednisone & $1.411(1.02,1.94)$ & 0.07637 \\
\hline CIART & Circadian-associated repressor of transcription & Allergen & $0.2(0.15,0.26)$ & $1.67 \mathrm{E}-14$ \\
\hline \multirow[t]{2}{*}{ 100121748_TGl_at } & & 10 mg Prednisone & $0.846(0.61,1.16)$ & 0.37841 \\
\hline & & 25 mg Prednisone & $1.003(0.73,1.37)$ & 0.98593 \\
\hline ARNTL & Transcription factor in circadian rhythm & Allergen & $1.411(1.24,1.59)$ & 0.00007 \\
\hline \multirow[t]{2}{*}{ 100142502_TGI_at } & & 10 mg Prednisone & $0.971(0.82,1.14)$ & 0.76279 \\
\hline & & 25 mg Prednisone & $0.933(0.79,1.09)$ & 0.46334 \\
\hline CRY1 & Cryptochrome 1 & Allergen & $1.53(1.34,1.75)$ & 0.00001 \\
\hline \multirow[t]{2}{*}{ 100144351_TGl_at } & & 10 mg Prednisone & $0.913(0.78,1.06)$ & 0.32261 \\
\hline & & 25 mg Prednisone & $0.818(0.70,0.95)$ & 0.03696 \\
\hline PER1 & Period circadian clock 1 & Allergen & $0.365(0.30,0.44)$ & $2.08 \mathrm{E}-9$ \\
\hline \multirow[t]{2}{*}{ 100145189_TGl_at } & & 10 mg Prednisone & $0.921(0.71,1.18)$ & 0.57276 \\
\hline & & 25 mg Prednisone & $1.32(1.03,1.69)$ & 0.06239 \\
\hline
\end{tabular}

Abbreviations: $\mathrm{Cl}$, confidence interval; FDR, false discovery rate; IL, interleukin; NAC, nasal allergen challenge.

IL-13 Responders only, FDR $<0.04$, selecting from 987 genes. 
a

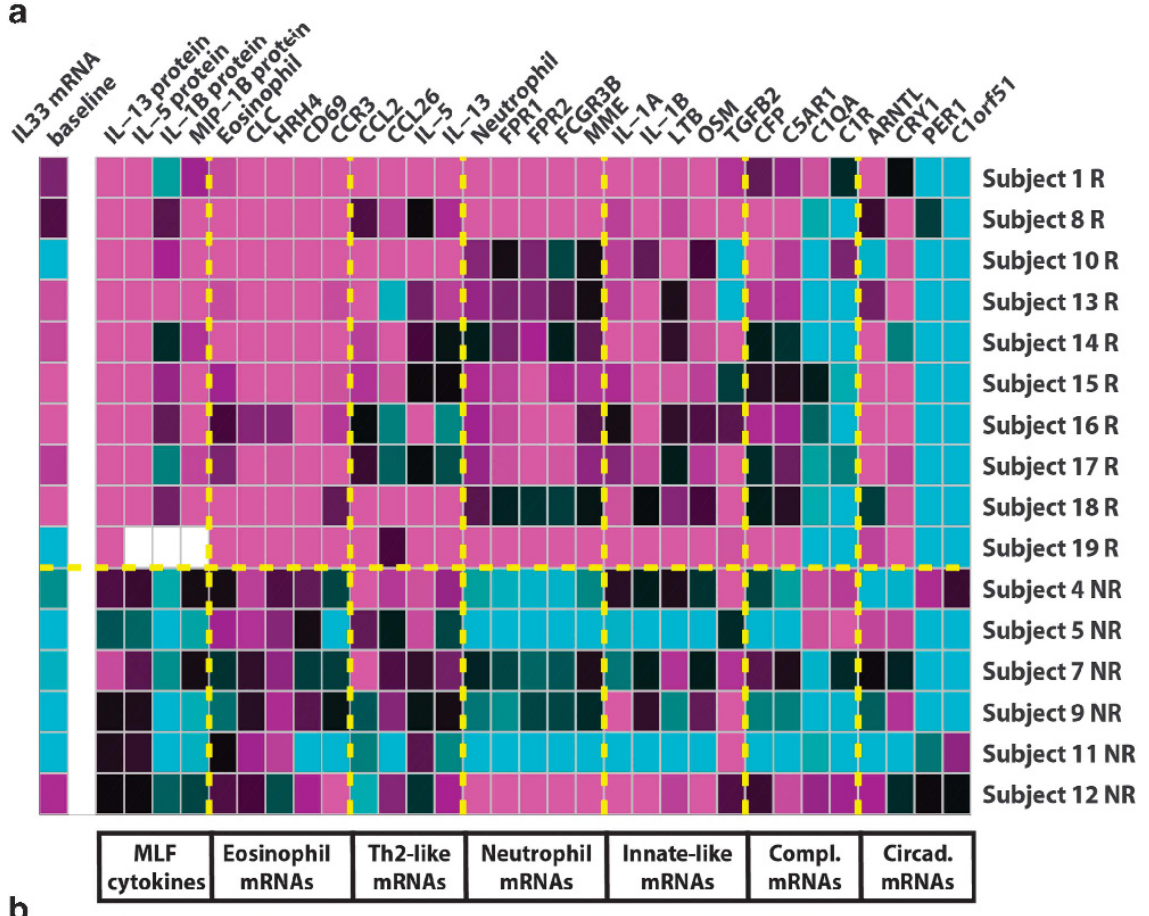

b

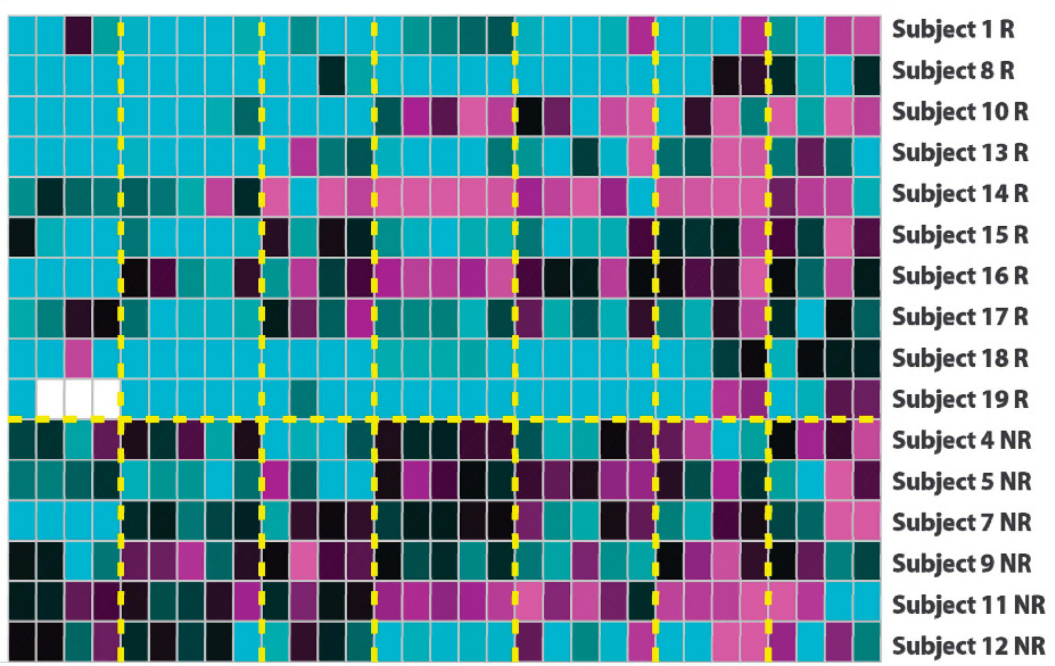

Figure 6 Heatmap representation of changes in expression of selected immune-related transcripts, mRNA signatures associated with eosinophils or neutrophils, and nasosorption cytokines. (a) Response to nasal allergen challenge (NAC) alone (upper) with baseline interleukin (IL)-33 mRNA expression appearing in a separate column on the left of a. (b) NAC preceded by single $25 \mathrm{mg}$ dose of prednisone (lower). Magenta represents upregulation, whereas cyan represents downregulation. "Eosinophil" and "neutrophil" refer to mRNA-derived cell-type composite markers (Supplementary Table E5 and Figure 3b). Horizontal dashed yellow line separates responders "R" from non-responders "NR". Vertical dashed yellow lines separate categories of analytes. White boxes signify missing values.

biomarkers. Having studied actions of a corticosteroid, this type of sampling approach with NAC could also be useful in the context of new drug development in early clinical phase human studies. In this way by studying a novel drug in a NAC design in a small well-defined population, it is possible to evaluate biomarkers and establish a pharmacodynamic profile.

The nasal LAR occurs in the majority of subjects (11 of 19) and is generally less distinct in terms of symptoms (TNSS) and physiological changes when compared with the late asthmatic reaction after inhaled allergen challenge, which can be precisely quantitated in terms of a decrease in forced expiratory volume in 1 second $\left(\mathrm{FEV}_{1}\right){ }^{23}$ The early nasal allergic reaction involves rapid onset of a range of nasal symptoms, but the late nasal response is generally due to partial nasal obstruction. The occurrence of peak symptoms in the first $30 \mathrm{~min}$ after NAC, with low levels of TNSS in the late phase, has been found by other investigators who also found that a single dose of topical nasal corticosteroid was found to have a minimal effect on symptom scores. ${ }^{24}$ However, in our study there were consistent trends for IL-13 responders to have more symptoms in the late 
phase and for a single dose of oral prednisone to decrease the level of these symptoms.

A weakness in our study is that TNSS and nasal function were not measured during the nasal early allergic reaction within the first hour of NAC. Nasal function can be measured by peak nasal inspiratory flow, ${ }^{14}$ acoustic rhinometry, or magnetic resonance imaging. ${ }^{25}$ In addition, it would have been of interest to have measured mediators during the early allergic reaction, such as tryptase, ${ }^{14} \mathrm{PGD}_{2}$ and IL-12/23p $40 .{ }^{26}$ Hence, it is not possible from the current study to assess the influence of symptoms, nasal function, and mediators during the early allergic reaction on the subsequent LAR. Indeed, mast cells are known to be major factors in the early allergic reaction and could have an important controlling influence on immune events in the LAR. Furthermore, it will be important to assess the clinical significance of having a LAR after NAC, both after $\mathrm{NAC}$ and in relation to natural exposure. However, given the weakness and variability of TNSS in the LAR, a larger sample size would be required for this endpoint.

Measuring the late nasal IL-13 response in MLF after NAC allowed the formal post-hoc identification of responder subjects (12 of 19). As a pre-specified primary endpoint was the GMTWA for IL-13, "responder" status was defined as nasal IL-13 GMTWA being significantly elevated compared with baseline levels. Responders had a nonsignificant trend for an increase in symptoms in the late phase. In addition, the biologic validity of selecting responders was supported by the concordance of IL-13 responses with those of IL-5 and IL- $1 \beta$, as well as with the first principal component across the allergen mRNA signature and post-NAC eosinophil-related gene signature levels.

A range of clinical studies have been carried out in severe eosinophilic asthma using biologics to inhibit type 2 inflammation. These have involved anti-IgE and monoclonal antibodies that block IL-4, 5, and $13 .^{18}$ In particular, recent studies with anti-IL-5 have selected patients for therapy based on a raised blood eosinophil count. ${ }^{27,28}$ Our study in patients with AR illustrates that raised IL- 5 in the LAR only occurs in some patients. This raises the question whether in patients with AR and allergic asthma there may also be considerable variation in levels of mucosal type 2 inflammation cytokines. In addition, we speculate that measurement of levels of cytokines in MLF, employing nasosorption and/or bronchosorption may assist in stratification of patients with a range of respiratory diseases and selection of patients for therapy with specific biologics.

In patients with AR it is logical to measure IgE responses at the target organ and much clinical research has been performed on local IgE production by the nasal mucosa. ${ }^{29}$ In our study, we did not measure IgE responses in nasosorption samples, although IgE has been measured in nasal lavage and nasal aspirates in $\mathrm{AR}^{30,31}$ In addition, it has been found that NAC can boost serum allergen-specific IgE at 4 weeks after a single NAC. ${ }^{32}$ Hence, following natural allergen exposure and after NAC with a range of natural and recombinant allergens, it would be of interest to measure nasal and serum total and specific IgE responses.
Following NAC, we used gene expression in nasal curettage samples to show prominent increases in an eosinophil signature, but also a concomitant influx of neutrophils. Neutrophil involvement in allergic reactions is well described and occurs at an earlier stage than eosinophil recruitment. Neutrophils are associated with cytokines such as IL- $1 \alpha$ and IL-1 $\beta$, and their recruitment may be partially mediated through IL-8. Collectively, this might reflect the presence on epithelial cells of patternrecognition receptors that can be activated by grass pollen, cause chemokine and cytokine production, and contribute to eosinophil and neutrophil recruitment in the LAR. ${ }^{33}$ Production of IL- $1 \alpha$ and IL- $1 \beta$ has been described in association with the nasal $\mathrm{LAR}^{34}$ following nasal lipopolysaccharide challenge; ${ }^{35}$ these events may be associated with the inflammasome. ${ }^{36}$ We were also interested to find elevation in IFNAR1 during the LAR, as this is an important regulator of IL-1 production and activation.

The alternative pathway of complement activation is a fundamental arm of the innate immune response to microbial pathogens. ${ }^{37}$ The current study showed upregulation of genes for the alternative pathway (CFP and C5aR1) and downregulation for genes of the classical pathway (especially genes for components of $\mathrm{C} 1$ ) following NAC. ${ }^{38}$ Complement $\mathrm{C} 3$ is expressed by bronchial epithelial cells ${ }^{39}$ and by mast cells, ${ }^{40}$ and cleavage by mast cell tryptase can generate anaphylatoxins (C3a and C5a) that can bind to mast cell receptors and continue this activation into the late phase. ${ }^{41}$ Indeed, our findings are consistent with prior reports of $\mathrm{C} 3 \mathrm{a}$ and $\mathrm{C} 5 \mathrm{a}$ detection after $\mathrm{NAC},{ }^{42}$ with increased nasal mucosal C3aR and $\mathrm{C} 5 \mathrm{aR}$ in $\mathrm{AR}^{43}$ and raised plasma $\mathrm{C} 3 \mathrm{a}$ and $\mathrm{C} 5 \mathrm{a}$ levels during allergen immunotherapy. ${ }^{44}$ Recently, it has been proposed that C3a and C5a may regulate type 2 and Th17 immune responses to allergens. ${ }^{45}$ Furthermore, when peripheral blood mononuclear cells from patients with AR are cultured with pollen allergens, there is dysregulation in genes of the complement system. ${ }^{46}$

NAC increases gene expression for protease-activated receptor-1 (thrombin receptor), suggesting an influence on coagulation. Thrombin levels have been shown to be elevated after NAC. ${ }^{47}$ Together, these features of the LAR suggest that grass pollen extract may act as a "microbial mimic" and trigger a diverse innate mucosal inflammatory response involving IL$1 \beta$ production with complement and coagulation activation.

This study also showed that baseline $I L-33$ gene expression in nasal curettage specimens taken from AR subjects at screening correlated strongly with the intensity of the subsequent LAR in the placebo arm after NAC. IL-33 is elevated on immunoassay of nasal secretions from patients with $\mathrm{AR}^{48}$ and polymorphisms in genes for the IL-33-IL1RL1 (IL-1 receptor-like 1) pathway have been associated with asthma. ${ }^{19,49}$ Importantly, IL-33 is found preformed in epithelial cells as an alarmin and is released by the nasal mucosa in response to stimuli such as mechanical contact, ${ }^{10}$ and IL-33 may be important in activating ILC2 cells. ${ }^{8}$

The fold-change induction of nasal IL-5 and IL-13 measured by immunoassay in this study were strongly correlated, suggesting a common cell of origin, perhaps by allergenspecific Th2 cells and/or ILC2 cells. ${ }^{8}$ In a recent study, flow cytometry was performed on nasal curettage dispersed cells and 
ILC2 cells were obtained before and after NAC with grass pollen. ${ }^{50}$ It was shown that allergic asthmatics had an enrichment of ILC2 cells at baseline and these numbers significantly increased after NAC, and correlated with peak IL-5 levels after NAC. However, it is still important to quantify the relative numbers of Th2 and IC2 cells that are responding to NAC. Thus, we hypothesize that baseline IL-33 expression reflects a rheostat-like mechanism that sets the response intensity of the mucosa to allergen exposure by regulating Th2 cells and/or ILC2 cells in the production of IL-5 and IL-13. If correct, this would extend the involvement of innate mechanisms in allergy and may offer further therapeutic options. ${ }^{8}$

Unexpectedly, circadian-associated regulatory transcripts were strongly modulated during the LAR. Circadian influences control corticosteroid production and molecular details of circadian-associated gene roles in immunity have been defined. ${ }^{51}$ More specifically, an epithelial circadian clock has been shown in Clara cells to control pulmonary inflammation and glucocorticoid action. ${ }^{52}$ In our gene expression analysis of the subjects with a LAR, we noted a profound differential effect on genes of the BMAL-1-CLOCK complex with upregulation of genes for activating factors such as BMAL-1, RORA, and RORC, whereas there was downregulation of genes for repressing factors such as PER1-3, Rev-ERB $\alpha$, and CIART. However, the strong decreases seen in PER1, CIART, and NR1d1 are very likely of epithelial origin, as downregulation in gene transcription by cells newly entering the sampled tissue are not likely to be reflected as strong mRNA reductions. Our clinical observations are consistent with recently described effects of epithelial circadian genes on inflammation in murine airway irritant models. ${ }^{52,53}$ Circadian gene expression changes were relatively weakly affected by prednisone pretreatment, suggesting that these may be useful targets for new therapy to complement corticosteroids-a hypothesis requiring testing in studies controlling for diurnal factors.

This study provides insights into the molecular basis of the nasal LAR to grass pollen. Using serial mucosal sampling, parallel inflammatory pathways were demonstrated and levels of inflammatory mediators were correlated with gene expression data. In addition, a dose response of a single dose of an oral glucococorticoid (prednisone) was demonstrated on cytokine and mRNA expression. The mixed immunopathology of the LAR has profound implications for therapy, as current antiallergic medications tend to be based on inhibiting type 2 inflammation. However, our results suggest potential roles of the inflammasome, complement activation, and circadianassociated genes in mediating immunopathology. A focus on these novel nasal mucosal pathways could potentially be used to select AR patients for rational specific therapy.

\section{METHODS}

Study design. We performed a randomized, double-blind, three-way crossover study to compare the effects of two single doses of oral prednisone (10 and $25 \mathrm{mg}$ ) with placebo on biomarkers of mucosal inflammation and transcriptomics post NAC (Figures 1a and b). All recruitment and study procedures were timed to occur outside the summer grass pollen season. Subjects were required to have a history of seasonal AR to grass pollen and also positive intra-epidermal skin prick tests to Timothy grass pollen extract. Patients with a history of other allergic diseases including asthma, eczema, and food allergy were excluded from the study. Blood eosinophil counts were measured at baseline in all subjects. Total and specific serum IgE levels were not measured. The study was registered with ClinicalTrials.gov as NCT0082806 and was overseen by the Research Ethics Committee of Hounslow and Hillingdon (London, UK).

Nasal allergen challenge. Timothy grass pollen (Aquagen, Phleum pratense; ALK, Horsholm, Denmark) was administered using a nasal Bidose applicator (Aptar Pharma, Crystal Lake, IL). A single nasal spray $(100 \mu \mathrm{l})$ was given to each nostril in each challenge (5000SQ-U/nostril $=\sim 1 \mu \mathrm{g}$ per nostril of Phleum P5 (Phl p 5) allergen). Sampling occurred before NAC and hourly thereafter. This repeated nasal sampling does not cause spurious elevations in nasal cytokines and chemokines. $^{27}$

Total nasal symptom scores. TNSS were recorded before NAC and hourly thereafter for $8 \mathrm{~h}$. Nasal congestion, rhinorrhoea, itching, and sneezing were scored from 0 to $3(0=$ none, $1=$ mild, $2=$ moderate, and $3=$ severe symptoms).

Nasosorption: collection of MLF. Synthetic absorptive matrix was cut into $7 \mathrm{~mm} \times 30 \mathrm{~mm}$ strips with rounded ends (Accuwik Ultra, Pall Life Sciences, Portsmouth, UK). Accuwik Ultra is no longer available, but a CE-marked nasosorption kit is available from Hunt Developments (Midhurst, West Sussex). Two synthetic absorptive matrix strips were carefully placed back to back and inserted into the right nostril against the inferior turbinate of the lateral wall of the nasal cavity. Nasosorption was performed for $2 \mathrm{~min}$, with application of nasal clips, to ensure good apposition on the mucosal surface of the nasal cavity. After withdrawal, synthetic absorptive matrix strips were placed into $300 \mu$ l of pre-chilled (4 C) assay buffer (Millipore AB buffer: containing protein, azide and detergent $\mathrm{pH}$ 7.4) preloaded into the upper chamber of a Spin-X cellulose acetate filtration device (Costar, Corning, NY). Spin filtration was then performed at $4{ }^{\circ} \mathrm{C}$ for $5 \mathrm{~min}$ at $16000 \mathrm{~g}$. The eluate was collected and immediately stored at $-80^{\circ} \mathrm{C}$ for later immunoassay.

Nasal lavage. This was performed using sterile normal saline $(5.0 \mathrm{ml})$ with flushing into the nasal cavity 20 times over $\sim 1 \mathrm{~min} .{ }^{54}$ Recovered fluid was then centrifuged to obtain cells and a differential leukocyte count performed after staining a standard cytospin.

Measurement of chemokines and cytokines in MLF. Concentrations of CXCL5 (12.33), IL-1 $\beta$ (0.44), IL-4 (2.83), IL-5 (0.27), IL-8 (2.10), MCP-1 (1.24), MIP1 $\alpha$ (20.9), MIP-1 $\beta$ (4.1), and RANTES (4.75) were determined using a Luminex 10-plex bead-based assay (R\&D Systems, Minneapolis, MN) and those of IL-13 (0.25) using a single-plex platebased assay (Meso Scale Diagnostics, Rockville, MD). Respective lower limits of quantitation $\left(\mathrm{pg} \mathrm{ml}^{-1} / \mathrm{ml}\right)$ appear in parentheses.

Nasal mRNA collection and measurement. Nasal scrapes were collected using Rhino-Probe plastic nasal curettes (Arlington Scientific, Springville, UT), with a baseline sample at screening and samples at $8 \mathrm{~h}$ after each NAC (Figure 1). Cells were immediately lysed in Qiagen RLT buffer and stored at $-70^{\circ} \mathrm{C}$ until purification using the RNeasy system (Qiagen, Hilden, Germany). RNA quality was assessed using an Agilent Bioanalyzer (RNA 6000 Pico Kit, Agilent, Santa Clara, CA). All analyzed samples had 28S/18S rRNA ratios of $>0.75$ and $<3$, and an RNA Integrity Number of $>5$. Total RNA was amplified using the NuGEN Ovation Whole Blood Solution protocol (NuGEN, San Carlos, CA). Amplified biotin-labeled material was hybridized to a custom-designed Affymetrix array (Gene Expression Omnibus GEO reference GPL10379). All analyzed arrays had percent of detected probesets $>30 \%$. Expression level data were submitted to the US NCBI and are accessible at http://www.ncbi.nlm.nih.gov/geo/query/ acc.cgi?acc=GSE67200. 
Statistical analyses. GMTWAs across the $2-8 \mathrm{~h}$ post-challenge time period were compared among the treatments using a mixed analysis of variance model for a three-period crossover design. The model included fixed factors for sequence, treatment, and period, and a random effect for patients within sequence. The between-treatment fold differences were estimated as the exponent of the difference in least-square means from the model; $90 \%$ confidence interval (one-sided $\alpha=0.05$ ) were also obtained from the model. The within-treatment differences were estimated in a similar manner. Cytokine concentrations were analyzed after a log transformation and thus assessed by a fold change relative to baseline.

Sample size was that required for $>80 \%$ power to detect a 0.5 -fold mean reduction in NAC-induced IL-5. ${ }^{15}$ The R statistical programming environment was used for inferences, correlations, and plot generation involving cytokines and chemokines alone. SAS 9.2 (SAS Institute, Cary, NC) was used for statistical inference and mixed linear models on cytokines and mRNA data, whereas Matlab 7.11.2 (R2010b, MathWorks, Natick, MA) with Statistics Toolbox was used for profiling-related plot generation, clustering, and principal components analysis on profiling data.

Nasal cytokines and "Responder" status. The primary endpoint for nasal cytokines was the fold change from baseline in the GMTWA ${ }_{2-8 \mathrm{~h}}$. Data in this LAR phase after NAC was log-transformed for analysis. To define participants who responded to NAC with IL-13 protein production ("Responders" and "Non-Responders"), a post-hoc analysis was done to identify the magnitude of the nasal IL-13 response relative to baseline $(-1 \mathrm{~h})$. A threshold for IL-13 was calculated from our data at baseline, below which $95 \%$ of fold changes in an unchallenged population would be expected to fall. Values of IL-13 greater than this threshold were used to identify "Responders".

Prespecified mRNA expression analysis. Normalization was performed using RMA quantile normalization. Only probes with Affymetrix MAS5 $P<0.05$ in $\geqslant 50 \%$ of samples in any treatment group were analyzed. This left 31892 probesets for analysis, of the 52369 on the array. In general, statistical analysis was restricted to probesets showing fold changes $>1.5$. Probeset expression levels were log-transformed before statistical analysis using a mixed model appropriate for a three-period crossover design. The model had terms for baseline (nasal scrape mRNA collection up to 28 days before starting treatment periods) as covariate, fixed treatment and period effects, and random subject effects. When multiplicity was explicitly addressed, Benjamini-Hochberg FDR was set to $10 \%$.

Unsupervised clustering of mRNA expression. Hierarchical clustering of the 365 probe sets strongly responsive to allergen in the prespecified analysis (Supplementary Datafile S1) was performed using correlations between changes in expression ( $8 \mathrm{~h}$ after NAC versus study baseline) as the distance metric, with average linkage used for clustering. mRNA expression was compared with NAC-induced IL-13 expression. To minimize possible bias associated with foldchange filtering, only MAS-5 filtering was used-resulting in 2,693 MAS5-present probesets for this analysis.

Identification of eosinophil, neutrophil and mast cell gene markers. A large database containing gene expression from over 1,000 blood and 1,000 respiratory tissue samples was analysed, to identify clusters of genes consistently correlated with across samples. K-means unsupervised clustering based on Pearson's correlation distance of oblique component was employed. Manual curation on the basis of $r$ values and published functional biology of the various genes was used to develop non-overlapping gene sets for eosinophils, neutrophils, and mast cells (Supplementary Table E5). These gene sets could then be used to assess the presence and/or activity of a particular cell type.

Ingenuity pathway analysis. Gene expression changes after NAC were analysed using 52369 probe sets corresponding to 31892 genes. Multiplicity correction was based on Benjamini-Hochberg FDR. The fold change $( \pm$ ) was ranked by $Z$-score, with a FDR cutoff of $<0.05$.
Data and materials availability. Microarray data obtained in this study are available at the Gene Expression Omnibus of the US National Institutes of Health's National Center for Biotechnology Information with accession number GDS67200.

SUPPLEMENTARY MATERIAL is linked to the online version of the paper at http://www.nature.com/mi

\section{ACKNOWLEDGMENTS}

We thank Margaret Imrie at Respiratory Clinical Trials for excellent technical support. We are grateful to Chan Beals and Jim Bolognese, Liliana Delgado and Michael Tanen, and George Tokiwa, at Merck Research Laboratories, for scientific and operational guidance, validation and performance of the cytokine assays, and validation of the nasal scrape profiling approach, respectively. Kim Strohmaier at Merck Research Labs and Tanushree Tunstall at Imperial College are thanked for administrative support. This work was funded by Merck (M.S.D.). T.T.H. acknowledges support from the Imperial National Institute for Health Research (NIHR) Biomedical Research Centre (BRC), whereas P.L. acknowledges the NIHR BRC at Guy's and St Thomas's NHS Foundation Trust and King's College London.

\section{AUTHOR CONTRIBUTIONS:}

T.T.H., B.R.L. and M.K.R. wrote the protocol. H.C., N.C., and I.D.L. provided modifications. B.R.L. and T.T.H. were principal clinical investigators. N.C., H.C., and I.D.L. provided Sponsor operational and compliance support. L.N.C. and M.K.R. were Sponsor's medical monitors. G.C.N. and A.J.T. performed patient and laboratory procedures. R.M. developed the statistical plan and analyzed cytokine and symptom data. V.M., G.C., C.T., and T.T.H. analyzed profiling data. P.L. and T.T.H. provided Ingenuity analyses. G.C., L.N.C., B.R.L., P.L., V.M., T.T.H., M.K.R., and C.T. interpreted the data. L.N.C. and T.T.H. wrote the first draft of the manuscript and B.R.L., P.L., V.M., and R.M. contributed to subsequent drafts and final manuscript. All authors reviewed the manuscript.

\section{DISCLOSURE}

H.C., L.N.C., G.C., N.C., I.D.L., R.M., V.M., M.K.R., and C.T. are or were MSD employees and may own stock or stock options in Merck. B.R.L. and G.C.N. are employees of Respiratory Clinical Trials. There are no patents arising from these data. All other authors declared no conflict of interest.

c) 2017 Society for Mucosal Immunology

\section{REFERENCES}

1. Hansel, T.T., Johnston, S.L. \& Openshaw, P.J. Microbes and mucosal immune responses in asthma. Lancet 381, 861-873 (2013).

2. Gauthier, M., Ray, A. \& Wenzel, S.E. Evolving concepts of asthma. Am. J. Respir. Crit. Care Med. 192, 660-668 (2015).

3. Wheatley, L.M. \& Togias, A. Clinical practice. Allergic rhinitis. N. Engl. J. Med. 372, 456-463 (2015).

4. Bourdin, A., Gras, D., Vachier, I. \& Chanez, P. Upper airway 1: allergic rhinitis and asthma: united disease through epithelial cells. Thorax 64, 999-1004 (2009).

5. Galli, S.J. \& Tsai, M. IgE and mast cells in allergic disease. Nat. Med. 18, 693-704 (2012).

6. Choy, D.F. et al. Gene expression patterns of Th2 inflammation and intercellular communication in asthmatic airways. J. Immunol. 186, 18611869 (2011).

7. Peters, M.C. et al. Measures of gene expression in sputum cells can identify TH2-high and TH2-low subtypes of asthma. J. Allergy Clin. Immunol. 133, 388-394 (2014).

8. McKenzie, A.N. Type-2 innate lymphoid cells in asthma and allergy. Ann. Am. Thorac. Soc. 11 (Suppl 5), S263-S270 (2014).

9. Alam, R., Sim, T.C., Hilsmeier, K. \& Grant, J.A. Development of a new technique for recovery of cytokines from inflammatory sites in situ. J. Immunol. Methods 155, 25-29 (1992).

10. Baumann, R. et al. Nasal levels of soluble IL-33R ST2 and IL-16 in allergic rhinitis: inverse correlation trends with disease severity. Clin. Exp. Allergy 43, 1134-1143 (2013). 
11. Baumann, R. et al. Comparison of the nasal release of IL-4, IL-10, IL-17, CCL13/MCP-4, and CCL26/eotaxin-3 in allergic rhinitis during season and after allergen challenge. Am. J. Rhinol. Allergy 27, 266-272 (2013).

12. Chawes, B.L. et al. A novel method for assessing unchallenged levels of mediators in nasal epithelial lining fluid. J. Allergy Clin. Immunol. 125, 13871389 (2010).

13. Folsgaard, N.V., Chawes, B.L., Bonnelykke, K., Jenmalm, M.C. \& Bisgaard, H. Cord blood Th2-related chemokine CCL22 levels associate with elevated total-IgE during preschool age. Clin. Exp. Allergy 42, 15961603 (2012)

14. Scadding, G.W. et al. Optimisation of grass pollen nasal allergen challenge for assessment of clinical and immunological outcomes. J. Immunol. Methods 384, 25-32 (2012).

15. Nicholson, G.C. et al. The effects of an anti-IL-13 mAb on cytokine levels and nasal symptoms following nasal allergen challenge. J. Allergy Clin. Immunol. 128, 800-807 (2011).

16. Proud, D. et al. Gene expression profiles during in vivo human rhinovirus infection: insights into the host response. Am. J. Respir. Crit. Care Med. 178, 962-968 (2008).

17. Poole, A. et al. Dissecting childhood asthma with nasal transcriptomics distinguishes subphenotypes of disease. J. Allergy Clin. Immunol. 133, 670-678 (2014).

18. Chung, K.F. Targeting the interleukin pathway in the treatment of asthma. Lancet 386, 1086-1096 (2015).

19. Moffatt, M.F. et al. A large-scale, consortium-based genomewide association study of asthma. N. Engl. J. Med. 363, 1211-1221 (2010).

20. Trompette, A. et al. Allergenicity resulting from functional mimicry of a Tolllike receptor complex protein. Nature 457, 585-588 (2009).

21. Roschmann, K.I. et al. Timothy grass pollen extract-induced gene expression and signalling pathways in airway epithelial cells. Clin. Exp. Allergy 41, 830-841 (2011).

22. Blume, C. et al. Barrier responses of human bronchial epithelial cells to grass pollen exposure. Eur. Respir. J. 42, 87-97 (2013).

23. Diamant, Z. et al. Inhaled allergen bronchoprovocation tests. J. Allergy Clin. Immunol. 132, 1045-1055 (2013).

24. Linden, M. et al. Immediate effect of topical budesonide on allergen challenge-induced nasal mucosal fluid levels of granulocyte-macrophage colony-stimulating factor and interleukin-5. Am. J. Respir. Crit. Care Med. 162, 1705-1708 (2000).

25. Leaker, B.R., Scadding, G., Jones, C.R. \& Barnes, P.J. Using magnetic resonance imaging to quantify the inflammatory response following allergen challenge in allergic rhinitis. Immun. Inflamm. Dis 3, 445-454 (2015).

26. Gunawardana, GC et al. The effect of vitamin D supplementation on mucosal II-5, MMP9 and cathelicidin after nasal allergen challenge with grass pollen. J Allergy Clin. Immunol. 137, AB73 (2016).

27. Bel, E.H. et al. Oral glucocorticoid-sparing effect of mepolizumab in eosinophilic asthma. N. Engl. J. Med. 371, 1189-1197 (2014).

28. Ortega, H.G. et al. Mepolizumab treatment in patients with severe eosinophilic asthma. N. Engl. J. Med. 371, 1198-1207 (2014).

29. De, S.E. et al. Local immunoglobulin e in the nasal mucosa: clinical implications. Allergy Asthma Immunol. Res 7, 321-331 (2015).

30. Benson, M., Reinholdt, J. \& Cardell, L.O. Allergen-reactive antibodies are found in nasal fluids from patients with birch pollen-induced intermittent allergic rhinitis, but not in healthy controls. Allergy 58, 386-392 (2003).

31. Sakaida, H., Masuda, S. \& Takeuchi, K. Measurement of Japanese cedar pollen-specific IgE in nasal secretions. Allergol. Int. 63, 467-473 (2014).

32. Niederberger, V. et al. Antigens drive memory lgE responses in human allergy via the nasal mucosa. Int. Arch. Allergy Immunol. 142, 133-144 (2007).

33. Lambrecht, B.N. \& Hammad, H. Allergens and the airway epithelium response: Gateway to allergic sensitization. J. Allergy Clin. Immunol. 134, 499-507 (2014).
34. Sim, T.C., Reece, L.M., Hilsmeier, K.A., Grant, J.A. \& Alam, R. Secretion of chemokines and other cytokines in allergen-induced nasal responses: inhibition by topical steroid treatment. Am. J. Respir. Crit Care Med. 152, 927-933 (1995).

35. Dhariwal, J. et al. Nasal lipopolysaccharide challenge and cytokine measurement reflects innate mucosal immune responsiveness. PLOS ONE 10, e0135363 (2015).

36. Latz, E., Xiao, T.S. \& Stutz, A. Activation and regulation of the inflammasomes. Nat. Rev. Immunol. 13, 397-411 (2013).

37. Holers, V.M. Complement and its receptors: new insights into human disease. Annu. Rev. Immunol. 32, 433-459 (2014).

38. Ricklin, D. \& Lambris, J.D. Complement in immune and inflammatory disorders: pathophysiological mechanisms. J. Immunol. 190, 3831-3838 (2013).

39. Varsano, S., Kaminsky, M., Kaiser, M. \& Rashkovsky, L. Generation of complement C3 and expression of cell membrane complement inhibitory proteins by human bronchial epithelium cell line. Thorax 55, 364-369 (2000).

40. Fukuoka, Y., Hite, M.R., Dellinger, A.L. \& Schwartz, L.B. Human skin mast cells express complement factors C3 and C5. J. Immunol. 191, 18271834 (2013).

41. Fukuoka, Y. et al. Generation of anaphylatoxins by human beta-tryptase from C3, C4, and C5. J. Immunol. 180, 6307-6316 (2008).

42. Andersson, M., Michel, L., Llull, J.B. \& Pipkorn, U. Complement activation on the nasal mucosal surface-a feature of the immediate allergic reaction in the nose. Allergy 49, 242-245 (1994).

43. Jun, S.W. et al. Overexpression of the anaphylatoxin receptors, complement anaphylatoxin $3 a$ receptor and complement anaphylatoxin $5 \mathrm{a}$ receptor, in the nasal mucosa of patients with mild and severe persistent allergic rhinitis. J. Allergy Clin. Immunol. 122, 119-125 (2008).

44. Bowser, C., Erstein, D.P., Silverberg, J.I., Nowakowski, M. \& Joks, R. Correlation of plasma complement split product levels with allergic respiratory disease activity and relation to allergen immunotherapy. Ann. Allergy Asthma Immunol. 104, 42-49 (2010).

45. Schmudde, I., Laumonnier, Y. \& Kohl, J. Anaphylatoxins coordinate innate and adaptive immune responses in allergic asthma. Semin. Immunol. 25, 2-11 (2013).

46. Couto, A.A. et al. Dysregulation of complement system and CD4 + T cell activation pathways implicated in allergic response. PLOS ONE 8, e74821 (2013).

47. Shimizu, S. et al. Role of the coagulation system in allergic inflammation in the upper airways. Clin. Immunol. 129, 365-371 (2008).

48. Asaka, D. et al. Elevated levels of interleukin-33 in the nasal secretions of patients with allergic rhinitis. Int. Arch. Allergy Immunol. 158: (Suppl 1)) 4750 (2012).

49. Savenije, O.E. et al. Association of IL33-IL-1 receptor-like 1 (IL1RL1) pathway polymorphisms with wheezing phenotypes and asthma in childhood. J. Allergy Clin. Immunol. 134, 170-177 (2014).

50. Dhariwal, $J$ et al. Novel nasal sampling techniques identify ILC2s as important responders in asthma during nasal allergen challenge. Am J Resp Crit Care Med 193, A7546 (2016).

51. Scheiermann, C., Kunisaki, Y. \& Frenette, P.S. Circadian control of the immune system. Nat. Rev. Immunol. 13, 190-198 (2013).

52. Gibbs, J. et al. An epithelial circadian clock controls pulmonary inflammation and glucocorticoid action. Nat. Med 20, 919-926 (2014).

53. Hwang, J.W., Sundar, I.K., Yao, H., Sellix, M.T. \& Rahman, I. Circadian clock function is disrupted by environmental tobacco/cigarette smoke, leading to lung inflammation and injury via a SIRT1-BMAL1 pathway. FASEB J 28, 176-194 (2014).

54. Greiff, L., Pipkorn, U., Alkner, U. \& Persson, C.G. The 'nasal pool' device applies controlled concentrations of solutes on human nasal airway mucosa and samples its surface exudations/secretions. Clin. Exp. Allergy 20, 253-259 (1990). 